\title{
Classification of Electron Gun Operation Modes Using Artificial Neural Networks
}

\author{
N. ISIK ${ }^{a, *}$ AND A.H. ISIK ${ }^{b}$ \\ ${ }^{a}$ Mehmet Akif Ersoy University, Department of Science Education, Burdur, Turkey \\ ${ }^{b}$ Mehmet Akif Ersoy University, Department of Computer Engineering, Burdur, Turkey
}

\begin{abstract}
In electron collision experiments, the seven-element electron gun is commonly used to accelerate and focus an electron beam. The main operation modes of this experimental device are afocal, zoom and broad beam-modes. Each of these operation modes can be used for producing electron beam with desired diameter. In this study, the artificial neural network classification technique (ANN) is used for classification of electron gun operation modes depending on electrostatic lens voltages. For this purpose, we investigate the focusing condition for the first three-element lens. Other ANN is employed for the second four-element lens voltages to find the electron gun operation modes. A comprehensive training data is obtained from SIMION software which uses traditional ray-tracing method. ANNs are trained with this dataset. Moreover, performance evaluations are carried out to determine the classification power of ANNs. High performance values show that the ANN can easily categorize the operation mode of the electron gun as a function of lens voltages. The proposed approach may help to adjust electron gun voltages before collision experiments. It is believed that this study will be a model for the future research in electron collision systems. Network can be trained with experimental data for practical applications.
\end{abstract}

DOI: 10.12693/APhysPolA.129.628

PACS/topics: 41.85.Ne, 07.05.Rm, 84.35.+I, 07.05.Mh

\section{Introduction}

There is an emerging need for fine-variable electron guns in a number of applications including radar guns, cathode ray tubes, and many experimental designs [1-6]. For these purposes, electron guns have been successfully designed and operated with different methods $[2-4]$. These approaches of the gun design depend on many parameters, including the electrostatic lens voltages, electron beam current and radial size of the beam in the image point. Because of the large number of parameters, these computational methods of finding optimum data are generally time consuming.

It is a remarkable fact that recently artificial intelligence technique has achieved significant advantages in optimal design of electrostatic lenses [7-11]. Artificial intelligence is a mathematical invention that simulates human intelligence [12]. Classification technique of artificial neural network (ANN) is an excellent data mapper based on processing units named neurons. The working principle of this method is to map an input into a desired output. In present study, we have used the ANN technique to simulate optical properties of a sevenelement electron gun. This study is focuses on useful operation modes of seven-element electron gun for practical applications, such as the electron impact collision experiments. According to the beam size, three basic operating modes have been investigated: the broad-beam, focused beam and afocal beam modes. In proposed method, classification of operation modes is achieved by training

*corresponding author; e-mail: nimetok@hotmail.com artificial neural network using the training data matrix. The data matrix is generated by the beam diameter in image point corresponding to the lens voltage values of the seven-element electron gun. The training data are obtained from SIMION 8.1 ray-tracing program which uses finite-difference method [13-14]. We analyze blind data that are not previously used in input data to test the ANN method ability. The architecture of network is calibrated until the best classification performance is achieved. The application of ANN technique in the researches of electron gun is performed for the first time in this study, to our knowledge. The method proposed in this study has time advantage in finding optimal operation gun-modes.

The study is organized as follows. In Sect. 2, an explanation of a seven-element electron gun and the description of three basic operation modes of the gun are given briefly. The results and discussions are given in Sect. 3. Finally, conclusions are given in the last section.

\section{Methodology}

In practice, the seven-element electron gun can be used in many applications [2]. The electron beam generated by thermionic emission from a heated filament is accelerated and is focused using a seven-element electron gun. The view of the seven-element electron gun and the electron beam trajectory obtained from SIMION 8.1 simulation program is demonstrated in Fig. 1. Filament is housed in the first element of the gun $\mathrm{E}_{0}$. Electrons produced by the filament pass from the lens $\mathrm{E}_{0}$ to $\mathrm{E}_{1}$. According to the operation principle of the gun, the first three electrostatic lenses are used to focus the beam. Aperture $\mathrm{A}_{3}$ (with diameter of $0.5 \mathrm{~mm}$ ) within third electrostatic lens $\mathrm{E}_{3}$ determines the radial size of the beam 
in the focus point (first image point; FIP). It is marked as focused beam in Fig. 1.

For the second part of the gun, the electron beam formed by the first three-elements $\mathrm{E}_{0}-\mathrm{E}_{3}$ is considered as an object. The second four element lens configuration $\mathrm{E}_{4}-\mathrm{E}_{7}$ is used to obtain the beam with required diameter in the interaction region. In our design, the final element $\mathrm{E}_{7}$ is held at ground potential for the simplicity. We have trained the network considering beam diameter at a distance of $50 \mathrm{~mm}$ from the gun. Following these operation principles of the gun, different electron beams can be acquired with voltage ratios of a three- and a four-element lens configuration. There are three main categorized groups in these networks for focused-, afocaland broad beam operation mode. We defined the electron beam with a diameter smaller than $0.3 \mathrm{~mm}$ as the focused beam. The afocal beam is described as the beam with diameter from $0.3 \mathrm{~mm}$ to $1 \mathrm{~mm}$. The beam having a diameter larger than $1 \mathrm{~mm}$ is defined as the broad-beam. The general layout of the networks is shown in Fig. 2. First network is designed to find the focused-beam mode in FIP using the voltages of first three elements of the gun (Fig. 2a). Second network is trained to know the relationship between the potentials of the second fourelement lenses $\mathrm{E}_{4}-\mathrm{E}_{7}$ and beam size at the image plane (Fig. 2b).

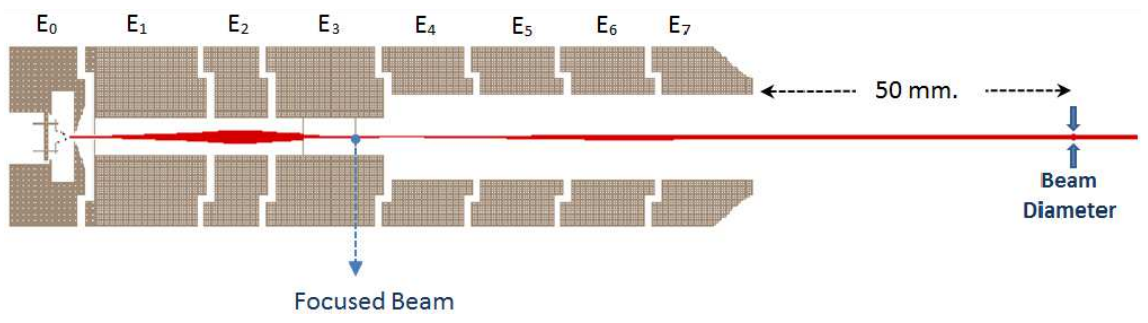

Fig. 1. View from the SIMION simulation program of the seven-element electron gun.
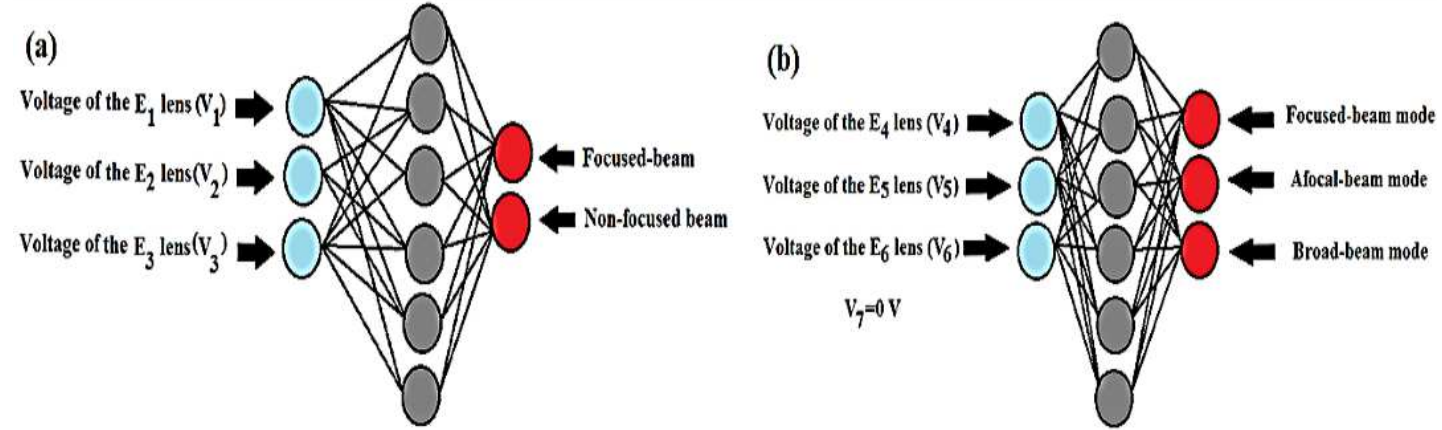

Fig. 2. The schematic view of architecture of (a) first and (b) second ANN that were used in this study.

\section{Results and discussion}

The main aim of this study is to obtain the lens voltages of electron gun for desired electron beam diameter using ANN model. The entire data set, obtained using the SIMION program, was filtered for an optimum level of expression. According to the gun operation, electron beam is firstly focused within $\mathrm{E}_{3}$ lens. After this, the desired beam diameters are obtained by changing the voltages of $\mathrm{E}_{4}-\mathrm{E}_{7}$ lens elements. In Fig. 3, beam diameters as functions of $\mathrm{E}_{4}$ lens voltage are presented. We used the simulation data set for $\mathrm{E}_{6}=\mathrm{E}_{7}=0 \mathrm{~V}, \mathrm{E}_{5}=5 \mathrm{~V}$ and $\mathrm{E}_{5}=15 \mathrm{~V}$. Three-operation modes depending on the beam size in the image plane are shown in this figure.

In ANN optimization, we have applied the scaledconjugated gradient algorithm [15]. Neural network toolbox in MATLAB/Simulink software package was used in this study. Input and output data of the network are divided into $70 \%$ training set, $15 \%$ validation set and $15 \%$ test set. The number of neurons in the hidden layer of the network was varied until the mean squared error had been minimized. Minimum mean squared error value indicates the best classification architecture. The results show that ANN has clearly separated the focusing voltages. "Over-training" of the model can be reached with increasing number of training epochs $($ Epoch $=20)$.

The classification performance is shown in Table I. In architecture with hidden layer composed of 8 neurons, the mean squared error was minimized for first classification. For second ANN, best classification performance in 3-20-3 architecture was obtained. Classification capabilities of these networks are tested with the blind test data set that was not used in the training process in 




Fig. 3. Training data set obtained from SIMION program. The electron beam size at $50 \mathrm{~mm}$ away from the last lens element as function of $\mathrm{E}_{4}$ voltage, for threeoperation modes. The potential of the sixth element lens is $\mathrm{E}_{6}=\mathrm{E}_{7}=0 \mathrm{~V}$.

advance. The classification performance of ANN is guaranteed with the small mean squared error values given in Table I.

TABLE I

Performance results of first and second ANN architecture.

\begin{tabular}{c|c|c|c|c|c}
\hline \hline \multicolumn{3}{c|}{ First ANN } & \multicolumn{3}{c}{ Second ANN } \\
\hline \multirow{3}{*}{ Architecture } & $\begin{array}{c}\text { Training } \\
\text { results }\end{array}$ & $\begin{array}{c}\text { Test } \\
\text { results }\end{array}$ & \multirow{2}{*}{ Architecture } & $\begin{array}{c}\text { Training } \\
\text { results }\end{array}$ & $\begin{array}{c}\text { Test } \\
\text { results }\end{array}$ \\
\cline { 2 - 3 } & MSE & MSE & & MSE & MSE \\
\hline $3-4-3$ & 0.172 & 0.199 & $3-4-3$ & 0.190 & 0.197 \\
$3-5-3$ & 0.142 & 0.151 & $3-5-3$ & 0.185 & 0.186 \\
$3-8-3$ & 0.101 & 0.131 & $3-8-3$ & 0.247 & 0.254 \\
$3-10-3$ & 0.188 & 0.259 & $3-10-3$ & 0.355 & 0.325 \\
$3-15-3$ & 0.210 & 0.174 & $3-15-3$ & 0.158 & 0.112 \\
$3-20-3$ & 0.182 & 0.184 & $3-20-3$ & 0.011 & 0.014 \\
$3-25-3$ & 0.200 & 0.216 & $3-25-3$ & 0.201 & 0.160
\end{tabular}

\section{Conclusions}

In this study, we have developed a method of optimization of seven-element electron gun using ANNs. Using SIMION program we have provided a minimal set that can correctly classify all data. Obtained results show that the ANN is a perfect tool for determining the operating mode of an electron gun with high-accuracy. We believe that the sensitivity of this method will be possible to improve with more experimental data. The ANN alternative method for determination of focusing characteristics of electron gun is developed in this study. The proposed method is advantageous for rapid response.

\section{Acknowledgments}

The authors would like to thank Omer Sise for helpful discussions.

\section{References}

[1] S. Raj, D. Sarma, Rev. Sci. Instrum. 75, 1020 (2004).

[2] M. Ulu, O. Sise, M. Dogan, Rad. Phys. Chem. 76, 636 (2007).

[3] B.M. Lewis, H.T. Tran, M.E. Read, R.L. Ives, IEEE Trans. Plasma Sci. 32, 1242 (2004).

[4] J. David, L. Ives, H. Tran, T. Bui, M. Read, IEEE Trans. Plasma Sci. 36, 156 (2008).

[5] T.W. Barefoot, H.D. Ebinger, J.T. Yates, J. Vac. Sci. Technol. A 15, 2740 (1997).

[6] S.K. Mahapatra, S.D. Dhole, V.N. Bhoraskar, Nuc. Instrum. Methods Phys. Res. A 536, 222 (2005).

[7] S.C. Johnston, W.A. Greynolds, D.Y. Wang, D.C. Dilworth, Proc. SPIE 1780, 192 (1993).

[8] S.M. Tam, C.K. Kwong, W.H. Ip, Int. J. Comp. Appl. Techn. 13, 229 (2000).

[9] A.A. Fadhil, Int. J. Adv. Comp. Tech. 2, 66 (2010).

[10] A.H. Isik, Acta Phys. Pol. A 127, 1317 (2015).

[11] A.H. Isik, Acta Phys. Pol. A 127, 1717 (2015).

[12] S. Haykin, Neural Networks: A Comprehensive Foundation, Prentice-Hall, New York 1999.

[13] Simion 3D v8.1, Scientific Instrument Services Inc., www. simion. com, 2012.

[14] D.W.O. Heddle, Electrostatic Lens Systems, IOP Press, London 2000.

[15] M.F. Moller, Neural Networks, 6, 525 (1993). 